\title{
Multi-Objective Optimization of the Safe Operation of the Electrical Distribution System by Placing D-FACTS and Network Reconfiguration
}

\section{Arouna Oloulade, Adolphe Moukengue Imano, Xavier Fifatin, Antoine Vianou, Herman Tamadaho, Ramanou Badarou}

Laboratoire d'Electrotechnique, de Télécommunications et d'Informatique Appliquée (LETIA), University of Abomey-Calavi, Abomey-Calavi, Benin

Email: olouarou@yahoo.fr

How to cite this paper: Oloulade, A., Imano, A.M., Fifatin, X., Vianou, A., Tamadaho, H. and Badarou, R. (2019) Multi-Objective Optimization of the Safe Operation of the Electrical Distribution System by Placing D-FACTS and Network Reconfiguration. Journal of Power and Energy Engineering, 7, 94-113.

https://doi.org/10.4236/jpee.2019.79007

Received: May 27, 2019

Accepted: September 24, 2019

Published: September 27, 2019

Copyright $\odot 2019$ by author(s) and Scientific Research Publishing Inc. This work is licensed under the Creative Commons Attribution International License (CC BY 4.0).

http://creativecommons.org/licenses/by/4.0/

\begin{abstract}
The distribution networks of agglomerated areas of the developing countries are generally the seat of overloads, voltage drops, and untimely interruptions of the power supply. This paper consisted of optimizing the grid topology and placement of a DSTATCOM in a SBEE real distribution network in order to improve its technical performance. The modified ant colony algorithms solved this difficult combinatorial problem, which integrated among the criteria, the minimization of the losses and the deviation of the node voltages under operational constraints about distribution networks operation. According to the results obtained, the optimization of the topology of a distribution network and the placement of DSTATCOM contributed qualitatively to improve the losses, voltage and stability plans of the Togba distribution network. Actually, the hybridization of optimization means such as the placement of DSTATCOM and the reconfiguration of the networks applied to the Togba HVA network made the power of DSTATCOM optimization possible by almost $50.71 \%$ and reduce losses to $83.57 \%$. The implementations of those algorithms are very efficient and effective, and can be implemented to help distribution system operators, developing countries and in particular, the operators of the Beninese Electric Power Company to perform their electrical network.
\end{abstract}

\section{Keywords}

Reconfiguration, Ant Colony, Electrical Stability, Radial Distribution Network 


\section{Introduction}

In the context of the liberalization of the energy sector, with the strong competition that follows it, the regulators have become very demanding in front of electricity companies. These requirements are driven not only by the urgent need to preserve fossil energy resources for future generations, but are also concerned about the quality of the product served to the consumer. Indeed, the electrical industry is subject to the laws and principles that govern both the physical characteristics of the product and the realization of the expectations of public services and consumers. The regulation is aimed at the general well-being that is the benefit of consumers and operators [1].

To achieve this, companies use several techniques to improve energy systems, including topology optimization, FACTS devices insertion, decentralized generation, placement of capacitors and inductors to control the transit of reactive and active power in the electrical networks. The hybridization of these means or the coupling of two different types of FACTS not only improves the technical performances but also contributes to reduce the computer drifts, the early convergences and the suboptimality of the solutions found [2].

Although the FACTS devices arising from the development of power electronics components, they constitute, nowadays, the effective means, to improve the technical performance of the transmission and distribution networks, their dimensioning and their location in the networks have led to other difficult optimization issues that network operators will have to face. In addition, in order to exploit the distribution networks, with greater profitability, the network operators use equipment such as SCADA and DMS that allow their automation and the control of all operating parameters. They consist among other functions in the automatic selection of the switches to be opened in order to minimize the active and reactive losses as much as possible and to supply healthy customers with a departure in case of occurrence of a disturbance. This complex function is part of the family of difficult optimization problems and whose metaheuristics provide global solutions, and can guide decision-makers to make optimal choices.

Moreover, the imbalance between reactive energy production and its consumption disrupts the maintenance of losses and voltage profiles within acceptable tolerances. In this context, the supply of good quality energy customers is one of the major problems of energy companies in order to meet the requirements of the system regulator and reliability and performance of their system. The solutions to this concern are often sought after by the authors who use metaheuristic methods to combine many actions to optimize the operational safety of electrical infrastructures.

In 2016, Radhika et al. [3] used cuckoo search algorithms (CSA) to reconfigure and place a FACTS in a distribution network. Watcharakorn Pinthurat \& al in 2018 [4], proposed an algorithm hybridization (PSO and AG) to optimize the operation of an electrical network by optimal positioning of a DG and reconfiguring it. Hajar Bagheri Tolabi \& al [5] proposed an ACO metaheuristic method 
associated with fuzzy logic to search the optimal position of a DSTATCOM, a photovoltaic cells in a distribution network and then reconfigure its topology. Venkateswarlu \& al [6] investigated the impact of a SVC and a DG on a distribution network and found that the optimal placement of a SVC and a DG significantly improves the ability of a transfer of an electrical network. JUNJIRO SUGIMOTO proposed in [7] the taboo search method to search the optimal position of a SVC and SVR in an electrical installation already constituted by a DG. M. A. Tayyab \& al. [8] show the effects of DG and SVC in a distribution network depending on their position. In this one, Yuvaraj \& al [9] proposed a metaheuristic based on the light search algorithm to optimize the placement of DSTAT$\mathrm{COM}$, and a DG in an electrical network, taking into account the variations of the load. Jai Govind Singh \& al [10] proposed another analytical method for optimal placement of a DSTATCOM and SVC to ensure the safety of the system in the event of sudden modifications in its characteristics. In 2013, Oloulade et al. [11] proposed a method for a DSTATCOM placement in a distribution network in order to improve the quality of customer supply. R. Sinirvassa [12] used bee colony to reconfigure the 33-bus network and found that this proposed method is efficient and effective. K. SURESHKUMAR et al. used the differential evolution technique to reconfigurate a distribution network.

This paper is devoted to optimizing the placement of a D-FACTS in a distribution network and reconfiguring its topology using a metaheuristic based on ant colony. The following part of the paper is constituted in point 2 by the driving difficulties faced by the operators, in 3 by the method used for the calculation of the power flow and the network stability, in 4 by the DSTATCOM modeling. Points 5, 6 and 7 present the ant colony algorithms, the simulation results and discussions then the conclusion.

\section{Operation Issues on Distribution Network of SBEE}

Electrical network operations are made of all the actions to be taken to maintain the supply of electrical energy at a good quality level and to effectively restore the electricity supply when it has been interrupted [13]. Actually, the detection and localization of defects, an essential component of the management of distribution networks, is done manually on the SBEE networks. The operations to be performed in case of occurrence of a defect are very long. Indeed, for simple disturbances and for a lack of sufficient information, the researches of dysfunctions are perilous. Operators are informed of faults on their facilities by telephone or radio from a service center or customers who have noticed the loss of voltage. After knowing their operating scheme, he proceeds to a series of actions on installing and affected equipment. This faults finding technique is very complex and becomes more complex when the fault occurs during peak hours. This research methodology induces very long intervention times and thus increases the undistributed energies. With regard to the development of the SBEE's distribution networks, it has been found that there is no coherent planning in cor- 
relation with the growth of the charges and their scale. There are, in fact, small sectorial projects aimed at the local reinforcement of certain installations which are often not very efficient and are not hierarchical. In fact, network planning will have to begin with an estimate of the demand to be served in the future, taking into account industrial and residential urban development plans, existing demand, and the impact of economic plans on growth. These shortcomings noted in the food system are the main causes of the surge in losses that it can't always master, and its difficulties in delivering good quality energy in some neighborhoods of large cities.

\section{Power Flow and Stability Analysis}

\subsection{Load Flow Analysis}

Distribution networks are characterized by high $\mathrm{R} / \mathrm{X}$ ratios and the traditional methods of power flow calculating widely known such as Newton Raphson, Gauss Seidel and Fast decoupled methods are not suitable for effectively solving the problems power flow problems in radial distribution networks.

The backward/forward sweep is one of load flow computation method which is developed and converges very well for radial distribution networks. It is based on the laws of ohm and Kirchhoff and can be considered from the family of iterative methods of power flow. This method is implemented on the Matlab platform and integrated into the optimal network reconfiguration algorithm [14].

\subsection{Stability Margin of a Distribution Network}

Voltage static stability in a distribution network has become an important concern for utilities today because of its impact on the quality and reliability of electricity supply. The companies then express the need to evaluate the stability of their system in order to predict the contingencies and to elaborate the means of safeguarding them. In the literature, there are several stability margin assessment methods, whose performances are variously appreciated by the authors. In this paper, we will use the continuous power flow method which consists of a reformulation of the power equations by integrating the load parameters to evaluate the stability margin of a HVA distribution network of the SBEE which is our case study.

$$
\begin{gathered}
\Delta P_{i}=P_{G i}(\lambda)-P_{L i}(\lambda)-P_{T i}=0 \\
\Delta Q_{i}=Q_{G i}-Q_{L i}(\lambda)-Q_{T i}=0
\end{gathered}
$$

with:

$$
\begin{aligned}
P_{T i} & =\sum_{j=1}^{n} V_{i} V_{j} Y_{i j} \cos \left(\theta_{i}-\theta_{j}-\gamma_{i j}\right) \\
Q_{T i} & =\sum_{j=1}^{n} V_{i} V_{j} Y_{i j} \sin \left(\theta_{i}-\theta_{j}-\gamma_{i j}\right)
\end{aligned}
$$

and:

$$
\Delta Q_{i}=Q_{G i}-Q_{L i}(\lambda)-Q_{T i}=0
$$


$\lambda=0$, correspond au cas de base.

$\lambda=\lambda_{\text {critique }}$ au cas critique.

The indices $L, G$ and $T$ respectively correspond to the load node, generation node (balance node) and injection node (PV node).

The $\mathrm{i}^{\text {th }}$ node voltage is $V_{i}\left\llcorner\delta_{i}\right.$ and $Y_{i j}\left\llcorner\gamma_{i j}\right.$ is the $(i, j)^{\text {th }}$ element of the $\left[Y_{\text {bus }}\right]$ system.

To simulate the different load variation cases, the power flow equations are modified as follows:

$$
\begin{aligned}
P_{L i}(\lambda) & =P_{L i o}+\lambda\left[K_{L i} S_{\Delta B a s e} \cos \left(\theta_{i}\right)\right] \\
Q_{L i}(\lambda) & =Q_{L i o}+\lambda\left[K_{L i} S_{\Delta B a s e} \sin \left(\theta_{i}\right)\right]
\end{aligned}
$$

where,

$$
\begin{gathered}
S_{\triangle B a s e} \cos \left(\theta_{i}\right)=P_{\text {Lio }} \\
S_{\triangle B a s e} \sin \left(\theta_{i}\right)=Q_{\text {Lio }} \\
P_{\text {Lio }} \tan \left(\theta_{i}\right)=Q_{\text {Lio }}
\end{gathered}
$$

Then, the equations above modified can be written:

$$
\begin{gathered}
P_{L i}(\lambda)=P_{L i o}\left[1+\lambda K_{L i}\right] \\
Q_{L i}(\lambda)=P_{L i o} \tan \left(\theta_{i}\right)\left[1+\lambda K_{L i}\right] \\
P_{G i}(\lambda)=P_{G i o}\left[1+\lambda K_{G i}\right]
\end{gathered}
$$

$P_{\text {Lio }}:$ Basic active power at $I^{\text {th }}$ node.

$Q_{\text {Lio }}$ : Basic reactive power at $I^{\text {th }}$ node.

$K_{L i}$ : Multiplier designating the proportion of variation of the load at node $i$.

$P_{G i}$ : Active power generated at node $i$ in initial state of system.

$K_{G i}$ : Constant specifying the proportion of generation variation when $\lambda$ change.

$S_{\triangle B a s e}$ : Apparent power chosen to provide an appropriate scale.

If " $F$ " is used as the set of equations, then, the system described above represents a set of nonlinear equations expressed by $F(x, \lambda)=0$ with $x=[\delta, V]^{\mathrm{T}}$ and it is applied the process of prediction and correction to solve these equations.

The calculation algorithm of the Continuous Power Flow (Algorithm 1) is essentially based on the prediction-correction method and is as follows:

Inputs: Node data ( $P, Q$, type), Line data $(R, X)$, Number of iterartion $N$.

Outputs: $V, \delta, \lambda$.

\section{Modeling of DSTATCOM}

The DSTATCOM is a device illustrated in Figure 1 and consists mainly of a voltage source continuously supplied and connected to the network through a transformer. The operating principle is to maintain a capacitor charged to the $V_{d c}$ DC voltage and to generate across the bridge a $V_{o}$ voltage using the energy stored in the capacitor and this voltage is in phase with the network voltage $\left(V_{S}\right)$. The difference between the amplitudes of the two voltages through 
Algorithm 1. Continuous Power Flow Algorithm

Step 1: Compute $V_{0}$ and $\delta_{0}$ to execute the algorithm described in subsection 2.4.2; $K=0$

Step 2: Reformulate the power flow equations to integrate the parameter $\lambda$ using Equations (4) - (7);

$$
\begin{aligned}
& \lambda=0 \\
& \text { As long as } d \lambda \neq 0 \text { and } k<N \text { do } \\
& K=k+1
\end{aligned}
$$

Step 3: Prediction of the new solution

Step 4: Calculate the tangent vector using the equation

Step 5: Predict the next solution based on the equation

Step 6: Fix the solution

Step 7: Correct the error on the predicted solution by solving the 2.32 system

Step 8: Choose the next continuous parameter based on the components of the tangent vector

$$
x_{k}: t_{k}=\max \left(t_{1} ; t_{2} ; t_{3} ; \cdots ; t_{m}\right)
$$

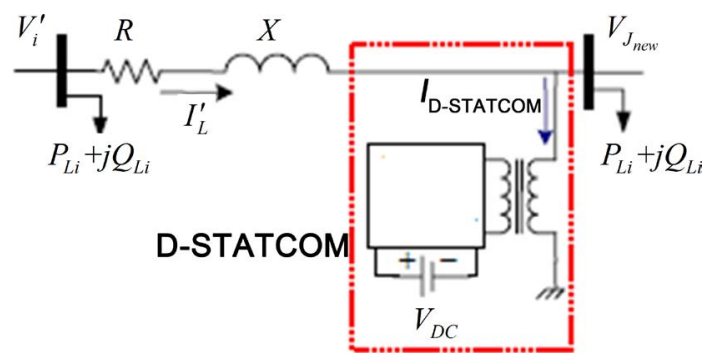

Figure 1. Diagram of a DSTATCOM.

the $X_{T}$ inductive reactance of the transformer creates the circulation of a reactive current.

The magnitude of this difference between the two voltages determines the amplitude of the reactive current. DSTATCOM is seen by the network as a reactive element of variable reactive current. This reactive current only depends on the amplitude of the $V_{o}$ voltage when the capacitor is charged. DSTATCOM can be used to perform compensation, control voltage, filter harmonics. In the power flow algorithm, the DSTATCOM is modeled as a current source that injects an alternative current into a distribution network.

Figure 2 shows the vector diagram of the two-node system.

For example, the head margin in this template measures proportionately more than is customary. This measurement and others are deliberate, using specifications that anticipate your paper as one part of the

$$
\begin{aligned}
& \arg \left(\overline{I_{\mathrm{D}-\mathrm{STATCOM}}}\right)=\alpha_{\text {new }}+\frac{\pi}{2} \\
& V_{J_{\text {new }}} \mathrm{e}^{j \alpha_{\text {new }}}=V_{i} \mathrm{e}^{j \theta}-(R+j X) I_{L}^{\prime} \mathrm{e}^{j \theta}-(R+j X) I_{\text {D-STATCOM }} \mathrm{e}^{j\left(\alpha_{\text {new }}+\frac{\pi}{2}\right)}
\end{aligned}
$$

With $I_{\text {D-STATCOM }} \mathrm{e}^{j\left(\alpha_{\text {new }}+\frac{\pi}{2}\right)}$ and $V_{J_{\text {new }}} \mathrm{e}^{j \alpha_{\text {new }}}$ which are respectively the injected 


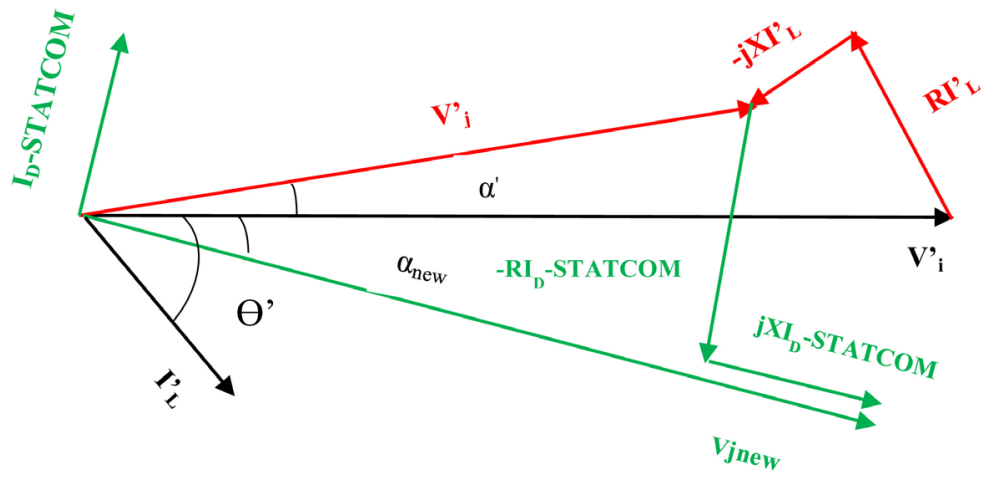

Figure 2. Vector diagram of the two-node system.

current by the DSTATCOM and the $j$ th node voltage after compensation.

By separating real parts and imaginary parts of Equation (10), we obtain the Equations (11) and (12):

$$
\begin{aligned}
V_{J_{\text {new }}} \cos \left(\alpha_{\text {new }}\right)= & \operatorname{Re}\left\{V_{i}^{\prime}-(R+j X) I_{L}^{\prime} \mathrm{e}^{j \theta}\right\}-R I_{\text {D-STATCOM }} \cos \left(+\frac{\pi}{2}\right) \\
& +X I_{\text {D-STATCOM }} \sin \left(+\frac{\pi}{2}\right) \\
V_{J_{\text {new }}} \sin \left(\alpha_{\text {new }}\right)= & \operatorname{Im}\left\{V_{i}^{\prime}-(R+J X) I_{L}^{\prime} \mathrm{e}^{j \theta}\right\}-R I_{\text {D-STATCOM }} \sin \left(+\frac{\pi}{2}\right) \\
& -X I_{\text {D-STATCOM }} \cos \left(+\frac{\pi}{2}\right)
\end{aligned}
$$

Data:

$$
\begin{gathered}
d=V_{j_{\text {new }}} \\
a=\operatorname{Re}\left\{V_{i}^{\prime} \angle \delta-(R+j X) I_{L}^{\prime} \angle \theta\right\} \\
b=\operatorname{Im}\left\{V_{i}^{\prime} \angle \delta-(R+j X) I_{L}^{\prime} \angle \theta\right\} \\
c_{1}=-R \\
c_{2}=-X
\end{gathered}
$$

The unknowns:

$$
\begin{gathered}
x_{1}=I_{\text {DSTATCOM }} \\
x_{2}=\alpha_{\text {new }}
\end{gathered}
$$

Then, the Equations (11) and (12) become respectively the Equations (13) and (14):

We can draw $x_{1}$ respectively in Equations (13) and (14):

$$
\begin{gathered}
x_{1}=\frac{d \cos x_{2}-a}{-c_{1} \sin x_{2}-c_{2} \cos x_{2}} \\
x_{1}=\frac{d \sin x_{2}-b}{c_{1} \cos x_{2}-c_{2} \sin x_{2}}
\end{gathered}
$$


Equalizing these two Equations (15) and (16) and then making a change of variable $x=\sin x_{2}$, we can observe, after an arrangement, the following:

$\left\{\left(a c_{2}-b c_{1}\right)^{2}+\left(a c_{1}+b c_{2}\right)^{2}\right\} x^{2}+2 d c_{1}\left(a c_{2}-b c_{1}\right) x+\left\{d^{2} c_{1}^{2}-\left(a c_{1}+b c_{2}\right)^{2}\right\}=0$

After that, we obtain:

$$
\begin{gathered}
\Delta=B^{2}-4 A C \text { avec } \\
A=\left(a c_{2}-b c_{1}\right)^{2}+\left(a c_{1}+b c_{2}\right)^{2} \\
B=2 d c_{1}\left(a c_{2}-b c_{1}\right) \\
C=d^{2} c_{1}^{2}-\left(a c_{1}+b c_{2}\right)^{2}
\end{gathered}
$$

We also obtain:

$$
x=\frac{-B \pm \sqrt{\Delta}}{2 A}
$$

where $x=\sin x_{2}$.

$x$ represents he initial conditions before the placement of DSTATCOM, the chosen Equation (19) is:

$$
x=\frac{-B+\sqrt{\Delta}}{2 A}
$$

Next,

$$
x_{2}=\arcsin (x)
$$

And we remind that $x_{2}=\alpha_{\text {new }} ; \alpha_{\text {new }}=x_{2} ; I_{\text {DSTATCOM }}=x_{1}$.

Then, $x_{1}=I_{\text {DSTATCOM }}$ is defined from Equations (15) or (16).

Then, we define:

$$
\begin{gathered}
V_{j_{\text {new }}}=V_{j_{\text {new }}} \angle \alpha_{\text {new }} \\
I_{\text {DSTATCOM }}=I_{\text {DSTATCOM }} \angle\left(\alpha_{\text {new }}+\frac{\pi}{2}\right)
\end{gathered}
$$

Finally, the reactive power injected by the D-STATCOM can be written as follows:

$$
j Q_{\text {DSTATCOM }}=V_{j_{\text {new }}} \times I_{\text {DSTATCOM }}^{*}
$$

where $I_{\text {DSTATCOM }}^{*}$ is the conjugate complex of the current of D-STATCOM.

The D-STATCOM is modeled so that the amplitude of the voltage of the node where it is installed is equal to $1 \mathrm{pu}$. The phase of this same node is computed from Equation (20), the current injected by D-STATCOM is calculated by Equation (22) and the reactive power injected is determined by Equation (23).

\section{Distribution Network Reconfiguration}

The search for a topology by the sequential opening of the branches (OSB) initially closed consists in successively opening appropriate branches. First of all, the network is completely meshed where all the branches are connected. To de- 
termine the branch to open, we compute the value of the objective function. The branch with the lowest loss value and the best network operating conditions will be open. This branch opening process is performed until an optimal exploitation scheme is obtained.

The algorithm developed for the search for optimal topology of a HVA network is based on the principle described as follows:

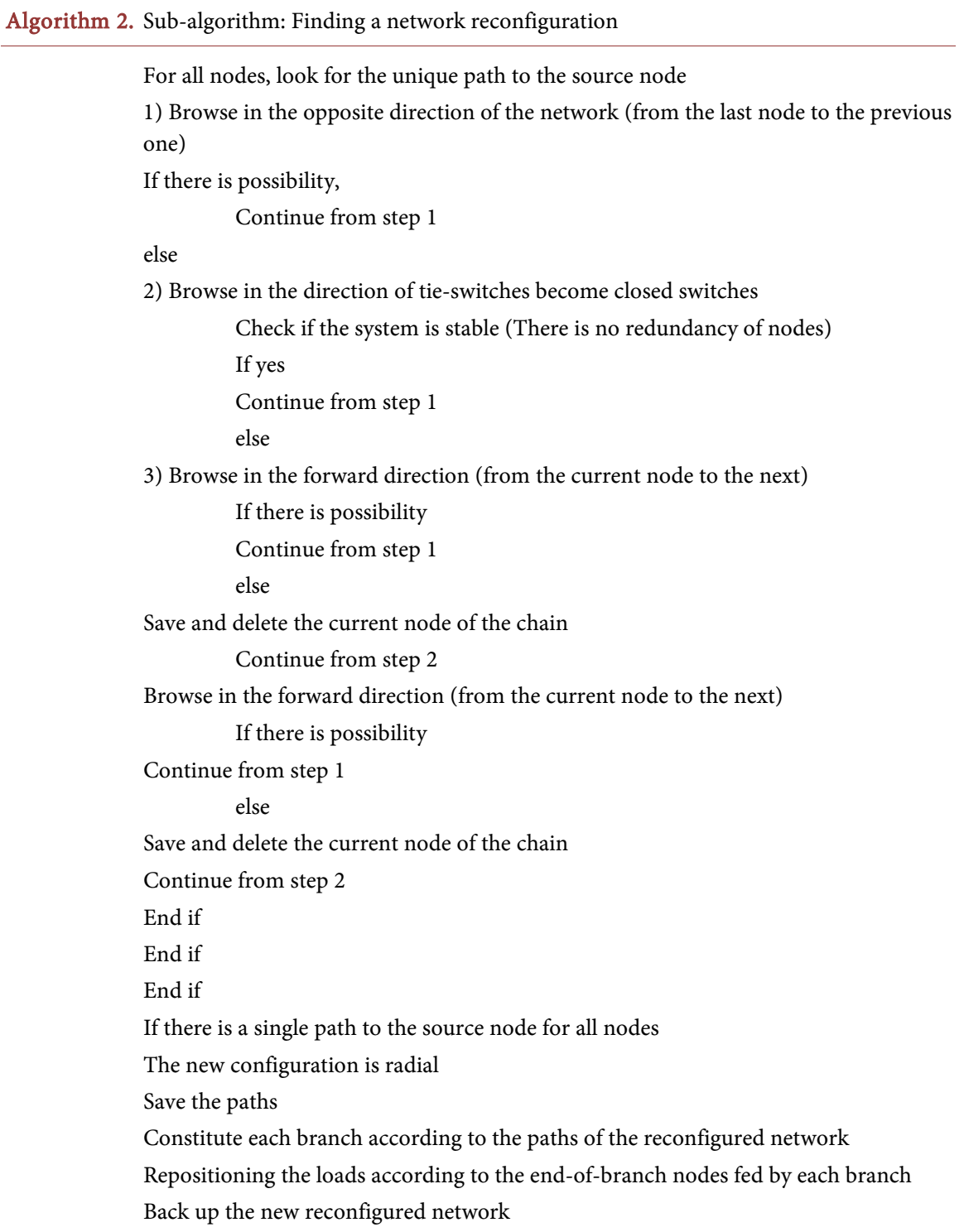

\section{Mathematical Formulation of the DSTATCOM Placement and the Topology Optimization Problems of a Distribution Network}

The optimizations of the placement of a DSTATCOM and the topology of a distribution network are a hybridization of two efficiency techniques of energy systems enhancement. It is a difficult combinatorial problem with conflicting objectives. 


\subsection{Objectives Functions}

- Losses minimizations

$$
f_{1}(x)=P_{\text {loss }}=\sum_{i=1}^{n_{b}} R_{i} \frac{P_{i}^{2}+Q_{i}^{2}}{V_{i}^{2}}
$$

With:

$R_{i}:$ resistance of branch;

$P_{i}$ : Active power of branch;

$Q_{i}$ : Reactive power of branch;

$V_{i}$ : Final node voltage of branch;

$n_{b}$ : branch number.

- Voltage deviation minimizations

The deviation of the voltage is given by:

$$
f_{2}(x)=D T=\sum_{i=1}^{N}\left(\frac{V_{i}-V_{i}^{\text {spec }}}{V_{i}^{\max }-V_{i}^{\min }}\right)^{2}
$$

$V_{i}: I^{\text {th }}$ node voltage and $V_{i}^{\text {spec }}$ its specific voltage;

$V_{i}^{\max }$ : maximum voltage which is $+0.5 \%$ of nominal voltage;

$V_{i}^{\text {min }}$ : minimum voltage which is $-0.5 \%$ of nominal voltage;

$N$ : number of overall nodes.

By aggregating the functions $f_{1}$ and $f_{2}$, we obtain the global objective function.

$$
\begin{aligned}
& F_{1}(x)=\alpha f_{1}(x)+\beta f_{2}(x) \\
& (\alpha, \beta) \in R^{2} \text { and }(\alpha+\beta)=1
\end{aligned}
$$

\subsection{Constraints}

$$
\begin{gathered}
V_{i}^{\min } \leq V_{i} \leq V_{i}^{\max } \\
P_{l}^{2}+Q_{l}^{2} \leq S_{l \max }^{2} \\
g_{2}=\frac{I_{i}}{I_{\max _{i}}}<1
\end{gathered}
$$

\section{Ant Colony Algorithm}

Ants are social insects whose physical or behavioral characteristics have long fascinated researchers. Ant colony algorithms typically use the behavior of real ants to solve combinatorial optimization problems. It can be reduced in search of the shortest path through graphs based on the behavior of the ants that in their displacement mark the way with glands contained in their abdomen called pheromones. The amount of pheromone depends on the length of the path and the amount of food found. The pheromone evaporates over time if others are deposited there. The path of pheromones leading to food sources will be more frequented by ants.

In practical terms, ant movements are characterized as a stochastic procedure 
for solutions around a graph such as $G=\{N, C\}$ where " $N$ " is the set of nodes and $C$ is the set of paths given by losses in the branches. Ants move according to a probabilistic decision rule are based on pheromone trails, ant condition and problem constants. The algorithm developed on the basis of the principles mentioned above is described as follows:

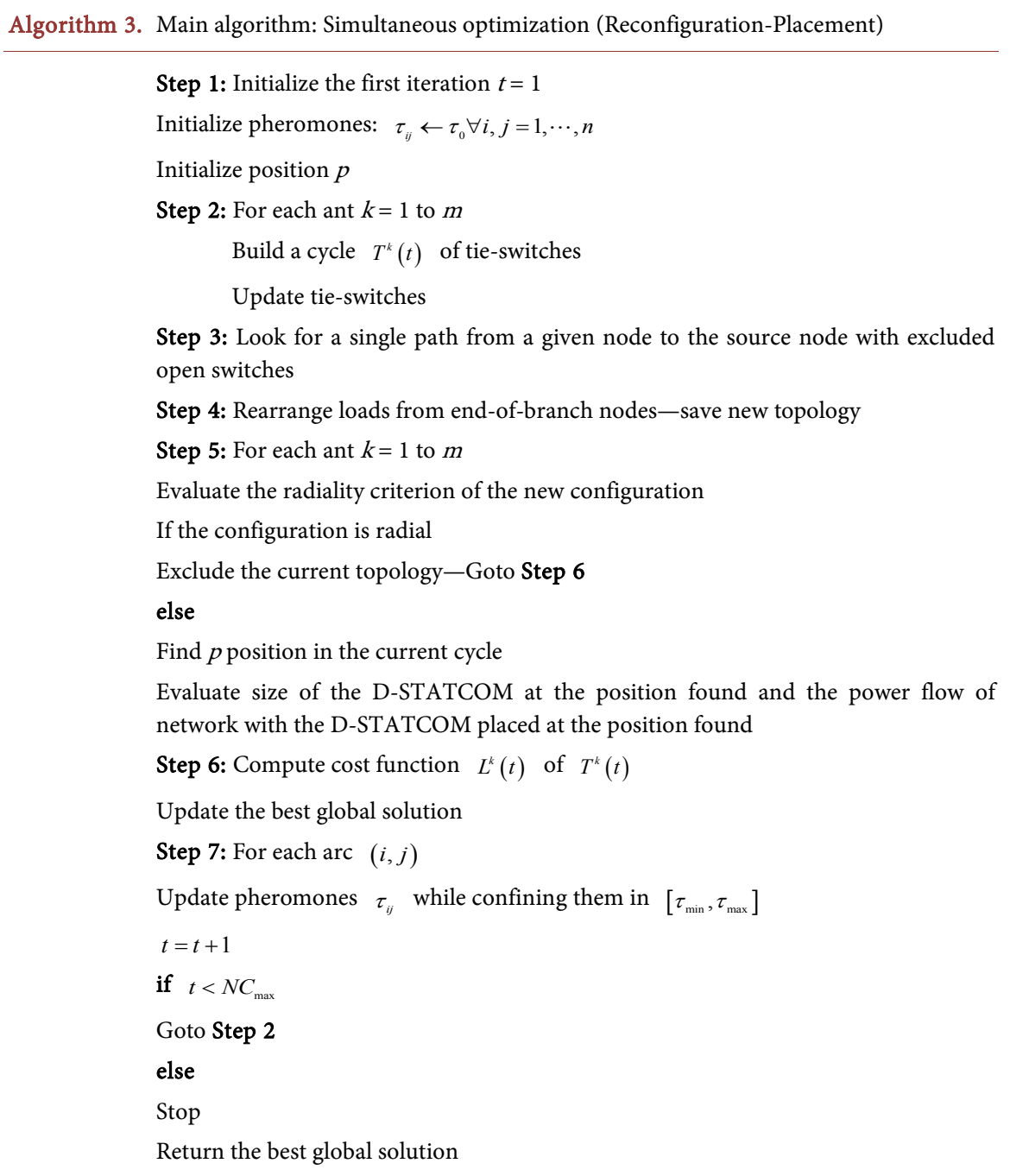

\section{Simulation Parameters}

The choice of parameter values determines the quality of solutions to be obtained in a simulation process. In fact, incorrect parameter settings or inappropriate choices can lead to suboptimal solutions. The table shows the values of the parameters chosen.

\section{Results and Discussions}

\subsection{Validation of the Results}

For validation purposes, we tested the tool designed under the MATLAB environment. For this purpose, it is tested on the standard 33-bus network. Figure 3 
shows the diagram of this network. It is composed of 33 nodes, 32 lines. The base voltage is $12.6 \mathrm{kV}$. The initially open switches are 33, 34, 35, 36 and 37 .

Table 1 presents the simulation results of reconfiguration and DSTATCOM placement in this network.

It is observed from Table 2 that the 33-bus network whose losses in the initial configuration are evaluated at $214.72 \mathrm{~kW}$ have been increased to $139.5 \mathrm{~kW}$ by using the differential evolution algorithm in [9] for the reconfiguration with switches 7, 9, 11, 32 and 37 opened versus 121, $56 \mathrm{~kW}$ by applying our method based on modified ant colonies. The switches that are open are 13, 10, 5, 27 and 18. The computer performance (computation time) is 18,331 seconds against 23 seconds that Nirmala found with his application.

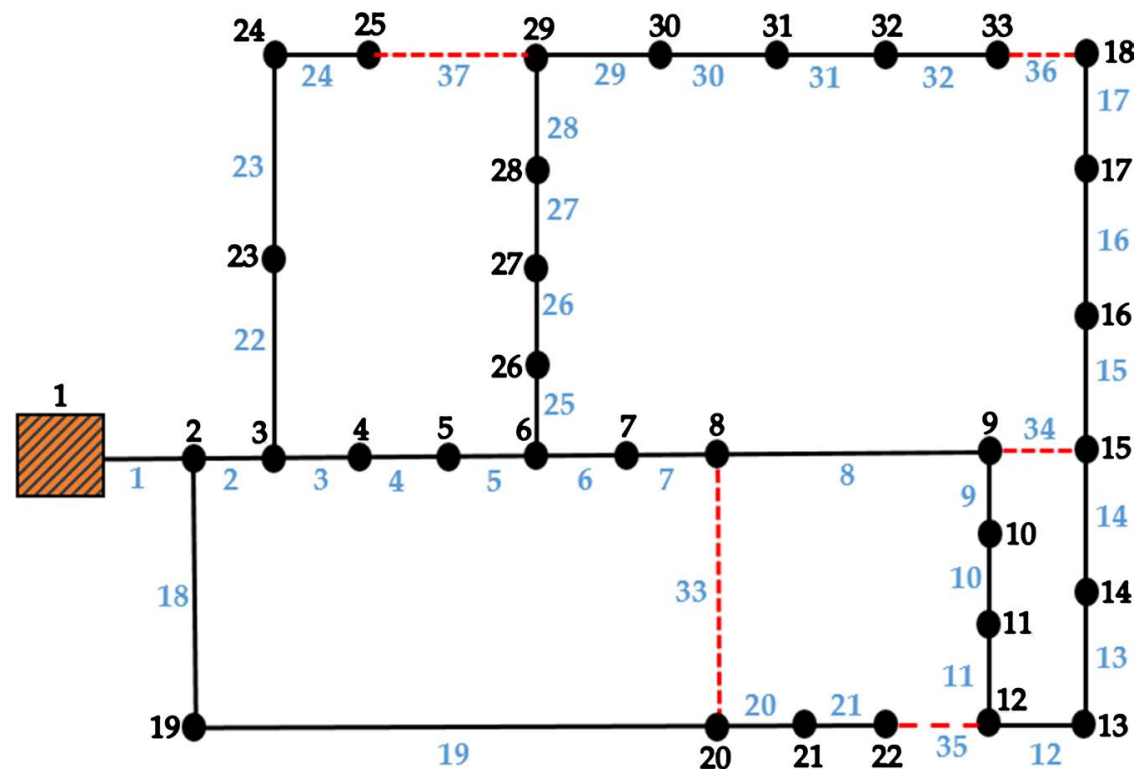

Figure 3. IEEE 33-bus network diagram.

Table 1. Simulation parameters.

\begin{tabular}{cc}
\hline Parameters & Values \\
\hline Colony size & 1 \\
Iterations number & 2 \\
$A$ & 100 \\
$B$ & 0.0005 \\
$Q$ & 0.7 \\
$\mathrm{pMin}$ & 0.05 \\
$\mathrm{pMax}$ & 0.7 \\
$\rho$ & 0.3 \\
$\lambda_{1}$ & 100,000 (high value) \\
$\lambda_{2}$ & 1 \\
$\Gamma$ & 2
\end{tabular}


Table 2. Validation of the results.

\begin{tabular}{|c|c|c|c|c|c|c|}
\hline & \multicolumn{4}{|c|}{ Reconfiguration } & \multicolumn{2}{|c|}{ DSTATCOM Placement } \\
\hline & Base configuration & $\mathrm{DE}[9]$ & $\mathrm{ABC}[10]$ & $\begin{array}{l}\text { Proposed Method } \\
\text { (ACO) [11] }\end{array}$ & VSI [11] & $\begin{array}{c}\text { Proposed Method } \\
\text { (ACO) }\end{array}$ \\
\hline Tie switches & $33,34,35,36,37$ & $7,9,11,32,37$ & $33,14,18,32,28$ & $13,10,5,27,18$ & - & - \\
\hline Actives losses & 214.72 & 139.5 & 139.5 & 121.56 & 143.03 & 137.68 \\
\hline$V_{\min }(\mathrm{pu})($ node $)$ & $\begin{array}{c}0.9128 \\
(28)\end{array}$ & $\begin{array}{c}0.9378 \\
(32)\end{array}$ & $\begin{array}{c}0.9437 \\
(33)\end{array}$ & $\begin{array}{c}0.9845 \\
(12)\end{array}$ & $0.9222 \mathrm{pu}$ & $0.9288 \mathrm{pu}$ \\
\hline Loss reduction (\%) & - & 31.2 & 35.03 & 43.38 & 33.38 & 35.87 \\
\hline $\mathrm{CPU}(\mathrm{s})$ & - & 23 & 5.3 & 18.331 & - & 5.563 \\
\hline DSTATCOM Size (MVar) & - & - & - & - & 3.386 & 3.1 \\
\hline DSTATCOM position & - & - & - & - & 30 & 30 \\
\hline
\end{tabular}

Sinirvassa applied the bee colony-based algorithm to reconfigure the 33 node network and found that the losses are also $139.5 \mathrm{~kW}$ and the switches to open are $33,14,18,32,28$. The computation time is $5.3 \mathrm{~s}$ compared to $18.331 \mathrm{~s}$ for the application of ACO to the same network. Based on the performance of the results, the algorithm developed can be applied to a real network of the SBEE in order to reconfigure the topology of an HVA departure.

\subsection{Application of ACO on the 41-Bus Network of SBEE}

The diagram of Figure 4 shows a HVA electrical system from a source station of Maria-Gleta (Benin). It consists of 41 nodes and normally open switches in basic operation.

Table 3 shows the results obtained by the simulation of the DSTATCOM placement and reconfiguration of the real network of SBEE. It is observed that the losses of the Togba network which were $403.56 \mathrm{~kW}$ for the basic configuration decrease to $66.30 \mathrm{~kW}$ after optimization by the placement of DFACTS and reconfiguration of the topology. It is noticed an improvement of $83.57 \%$.

The reconfiguration associated with the placement of DSTATCOM in a network has a positive impact on the technical performances of the distribution network. This action can make it possible to defer the investments required to reinforce the essential equipment of distribution networks such as transformers and lines whose reinforcement often requires a lot of financial resources. The optimal power of the DSTATCOM, in the case of simple placement, which is 2.1 MVar with a position at node 36 [11] is passed to node 20 with a power of 1.035 MVar. The power of DSTATCOM is then reduced by almost $50.71 \%$. Optimizing the topology of a distribution network associated with the placement of DSATCOM is then very economical and efficient and can further optimize the power of the compensator needed to improve the network.

The voltage, the lowest on the network that was $0.8498 \mathrm{pu}$ at node 37 for the basic configuration become $0.9510 \mathrm{pu}$ to node 32 for the simple placement case, 


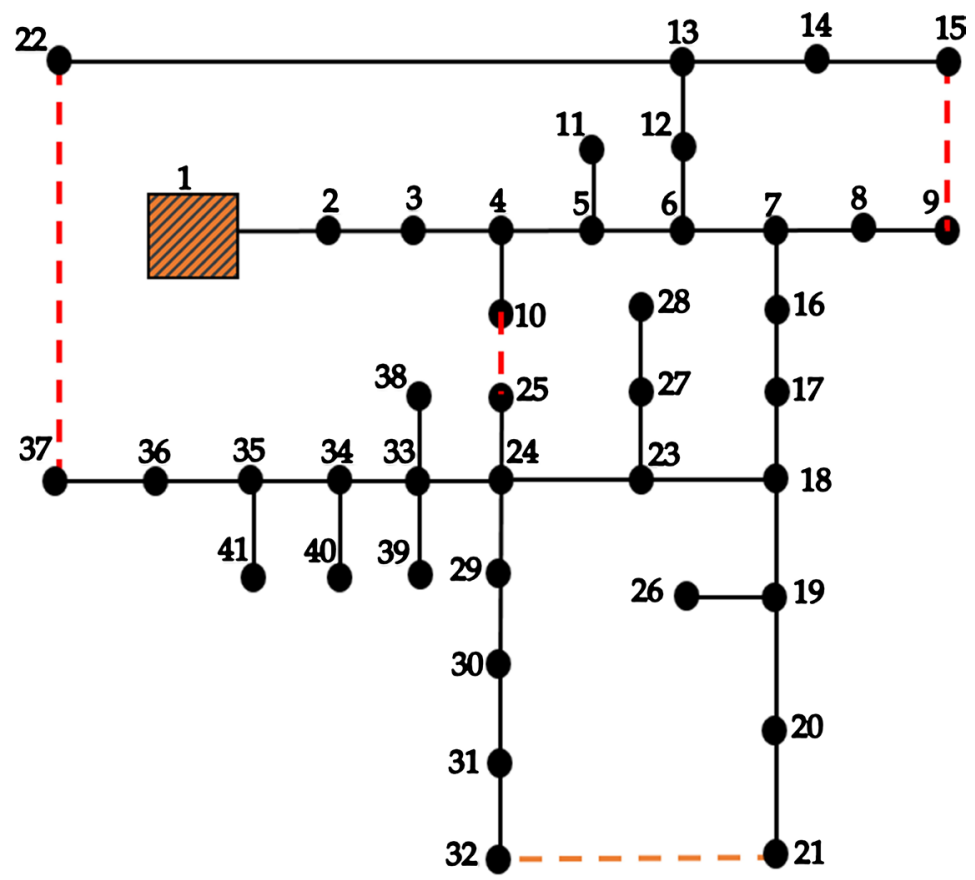

Figure 4. Network topology before reconfiguration.

Table 3. Summary of the results.

\begin{tabular}{ccccc}
\hline & $\begin{array}{c}\text { Base } \\
\text { configuration }\end{array}$ & $\begin{array}{c}\text { DSTATCOM } \\
\text { position }\end{array}$ & Reconfiguration & $\begin{array}{c}\text { DSTACOM + } \\
\text { Reconfiguration }\end{array}$ \\
\hline Lower voltage pu (node) & 0.8498 & 0.9510 & 0.9724 & 0.9886 \\
& $(37)$ & $(32)$ & $(23)$ & $(8)$ \\
DSTATCOM size (MVar) & - & 2.1 & - & 1.03 \\
DSTATCOM position & & 36 & - & 20 \\
Active losses (kW) & 403.38 & 164.77 & 148.97 & 66.30 \\
Losses reduction (\%) & & 59 & 63.08 & 83.57 \\
& $9-15 ;$ & $9-15 ;$ & $8-9 ;$ & $4-5 ;$ \\
Tie switches & $21-32 ;$ & $21-32 ;$ & $23-24 ;$ & $7-8 ;$ \\
& $10-25 ;$ & $10-25 ;$ & $30-31 ;$ & $21-32 ;$ \\
Stability margin (MW) & $22-37 ;$ & $22-37 ;$ & $35-36 ;$ & $37-22 ;$ \\
Margin improvement (\%) & -17 & 3.41 & 4.4 & 5.17 \\
\hline
\end{tabular}

$0.9724 \mathrm{pu}$ at node 23 for the reconfiguration and $0.9886 \mathrm{pu}$ at node 8 for the last scenario. It is observed that the voltage was better corrected for the case of reconfiguration than in the case of DSTATCOM placement of $2.20 \%$. Compared to the basic configuration, the placement of DSTATCOM associated with the topology reconfiguration not only optimizes the power of DSTATCOM required but also reduces losses economically and technically in very interesting proportions.

Figure 5 shows the voltage profiles on the busbar sets of the Togba HVA networks. It is observed that the voltage profile as a result of the reconfiguration 
is significantly improved than the voltage plane in the case of only DSTATCOM placement. Indeed, for the DSTATCOM placement case, several voltage values are below $0.96 \mathrm{pu}$, whereas for the case of reconfiguration, most of the voltage values are beyond $0.98 \mathrm{pu}$. The reconfiguration had more effect on the voltage profile than the DSTATCOM placement. Several problems related to voltage drop that affect the quality of supply and do not allow several customers to eat properly can be solved by optimizing the network topology. Designers of distribution networks already have to design the reconfigurable networks by considering the switches sized in accordance with the transit, and appropriate cable sections to facilitate the reconfigurations that must adapt to the load profile.

Figures 6-8 shows the PV voltage stability curves at nodes 4, 11 and 36 of the Togba electrical network. It can be seen that the stability margin for the base case is $2.1 \mathrm{MW}, 3.17 \mathrm{MW}$ with the placement case at node 36, $4.61 \mathrm{MW}$ with reconfiguration and 5.17 MW with simultaneous placement and reconfiguration. The stability margin of the basic configuration of $2.17 \mathrm{MW}$ shows that this network in this state is almost disastrous and has several neuralgic points and probable instability of voltage. In this state, it can no longer allow the connection of new loads at the risk of collapse. On the other hand, the margin of stability is improved after the various optimization scenarios. The point separating the operating point of the network from the collapse point is significantly improved with reconfiguration than the placement case by nearly $31.23 \%$. This margin of stability is further improved in the combination of the two network improvement actions. The increase of voltage stability after optimization may allow the addition of other loads to this network without the need to reinforce existing

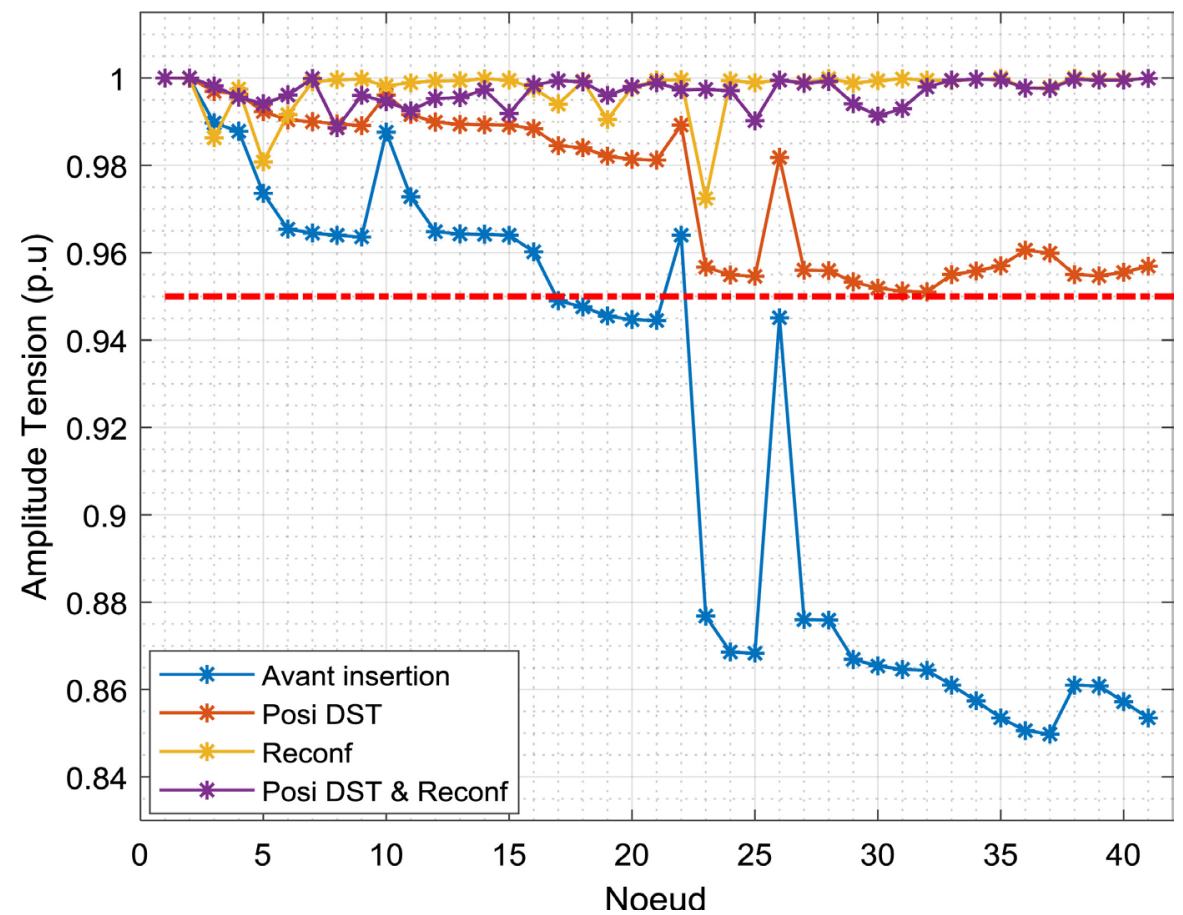

Figure 5. Voltage profile. 
Courbes P-V aux nœuds 4,11 et 36 après insertion du DSTATCOM

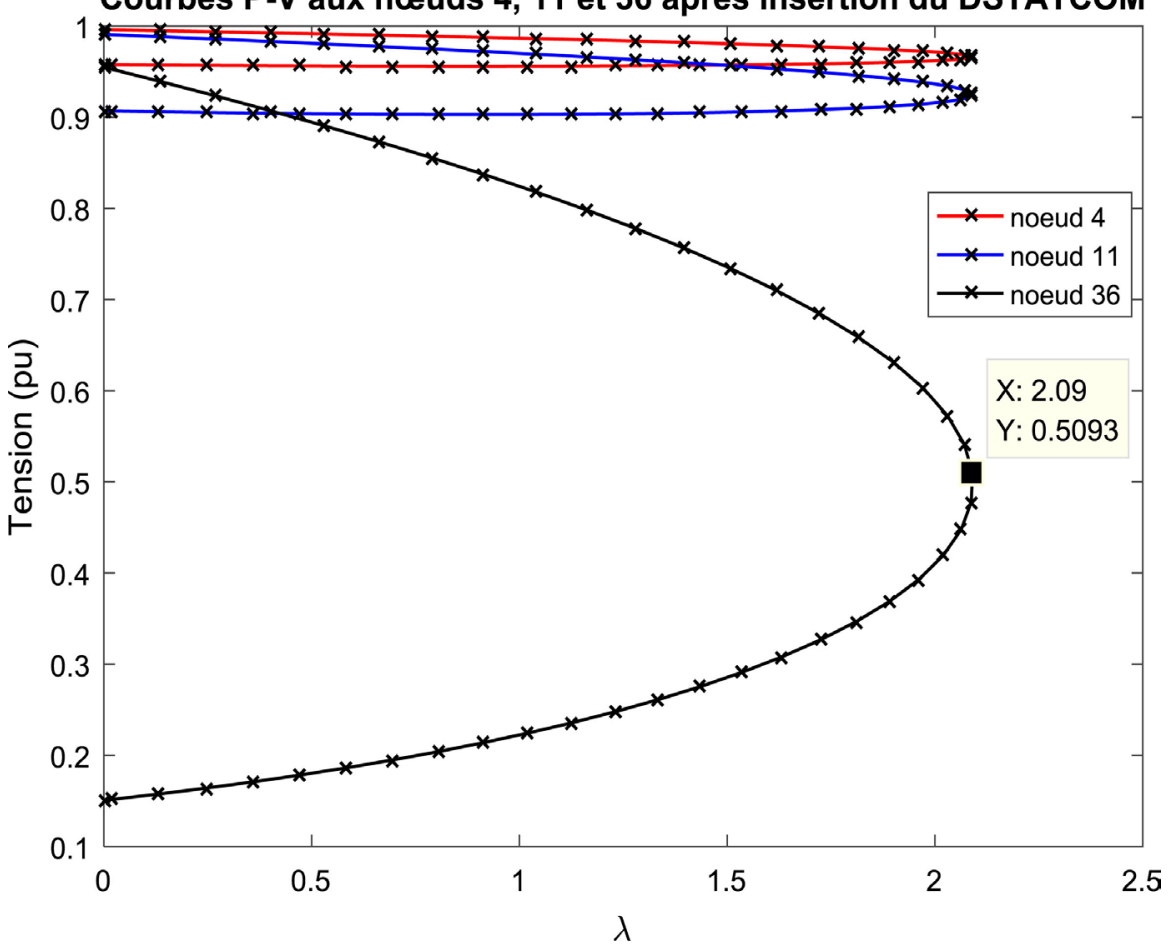

Figure 6. PV curves after DSTATCOM placement at nodes 4, 11 et 36 .

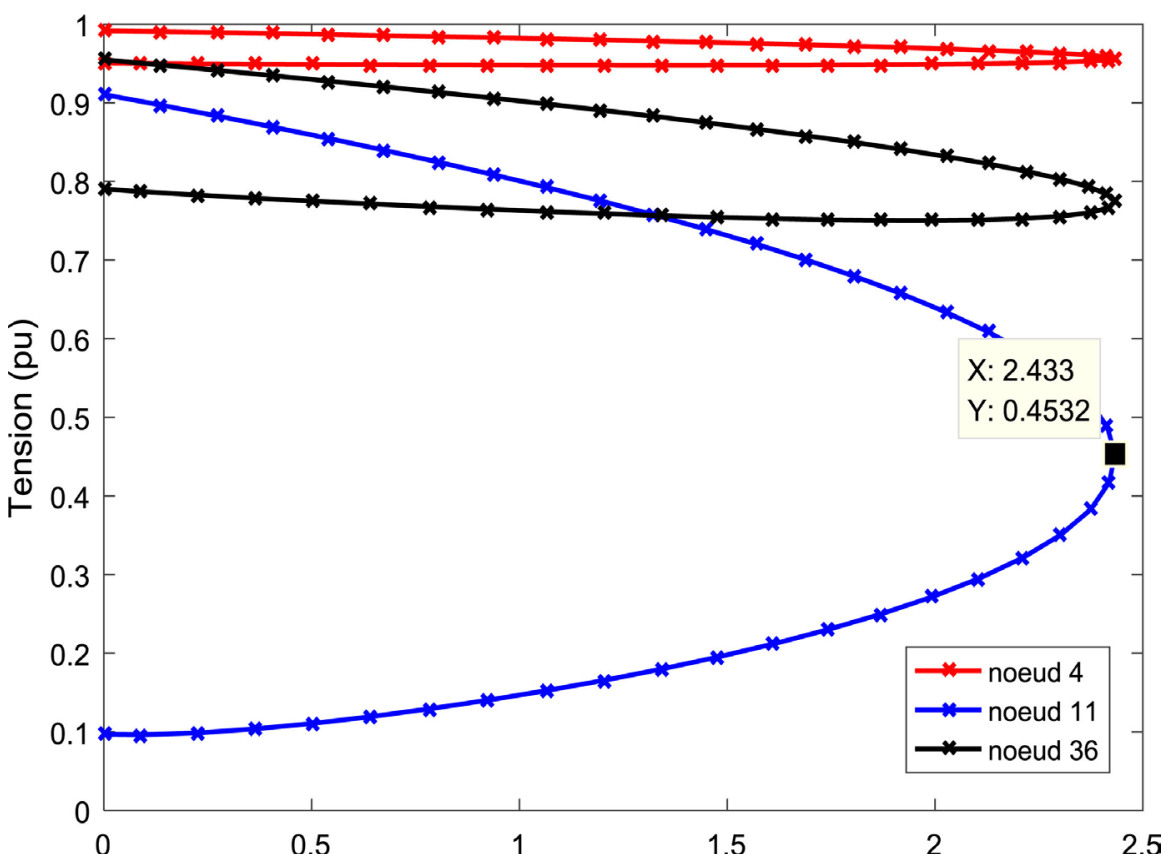

Figure 7. PV curves at nodes 4,11 and 36 after reconfiguration.

network equipment (lines and transformers). This optimization can then reduce the operation and maintenance costs of networks while strengthening their operational safety.

Figure 9 shows the topology of the reconfigured Togba network. The opened switches as a result of the reconfiguration are $8-9 ; 23-24 ; 30-31 ; 35-36$. Be- 


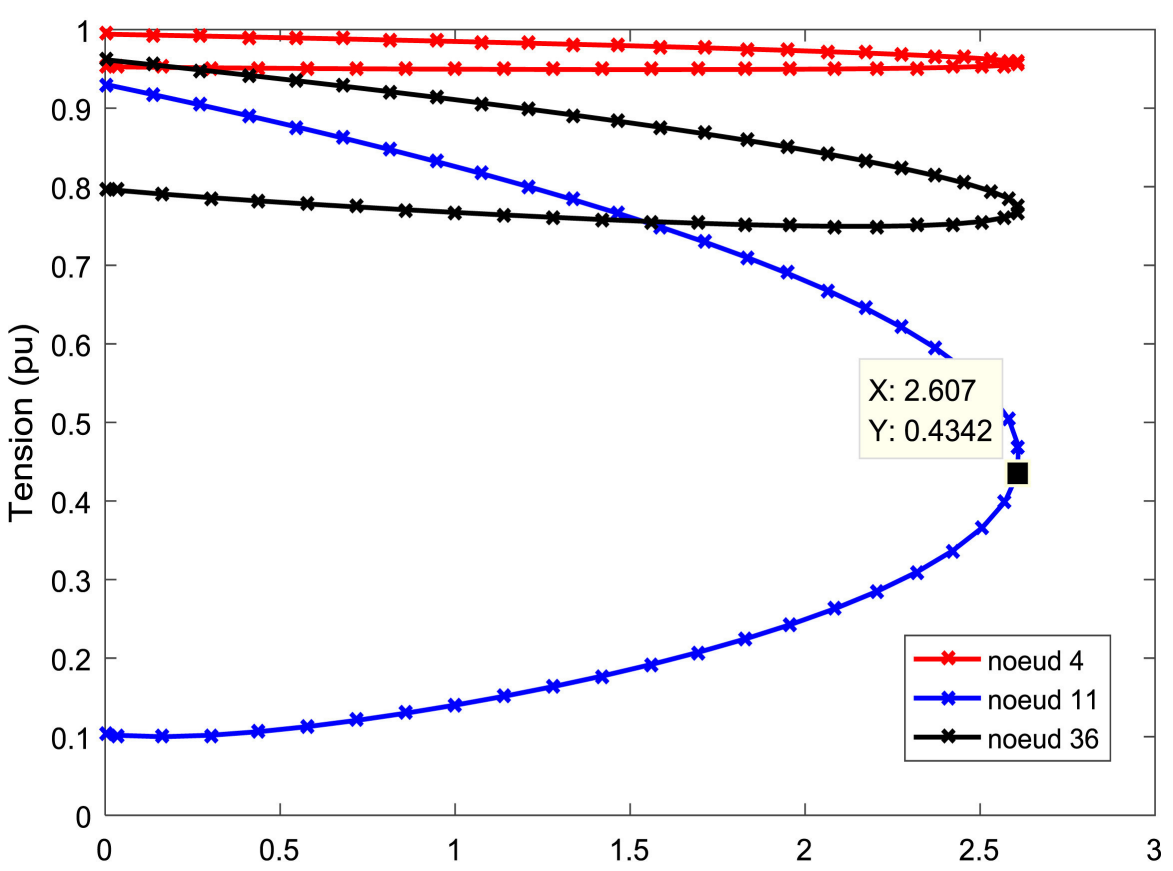

Figure 8. PV curves at nodes 4, 11 et 36 after DSTATCOM placement and reconfiguration.

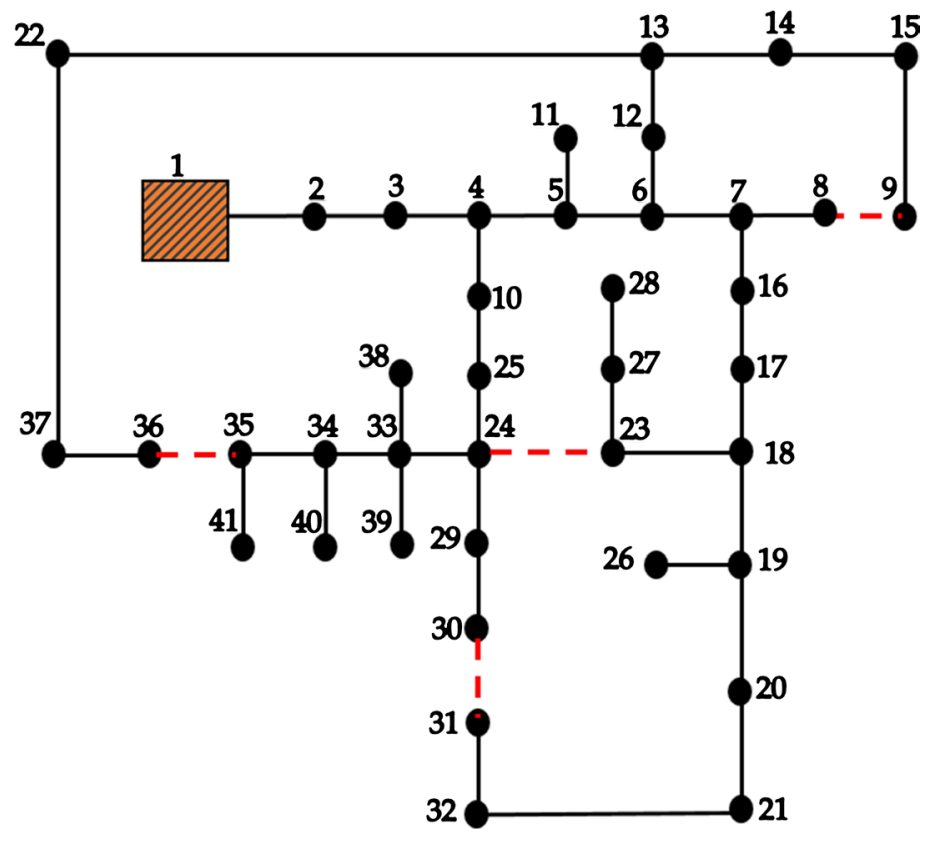

Figure 9. Network topology after reconfiguration.

sides, Figure 10 shows the results of simultaneous placement of DSTATCOM with the network reconfiguration. It is observed that the DSTATCOM is placed at $20^{\text {th }}$ node with a power of 1.03 MVar compared to the placement case where the node 36 is the position of DSTATCOM with a power of 2.1 MVar. Open switches went from 8 - 9; 23 - 24; 30 - 31; 35 - 36 (reconfiguration case only) to 4 - 5; 7 - 8; $21-32 ; 37-22$ (reconfiguration and placement simultaneously case) while providing power to all consumers. Then, it can be concluded that the re- 


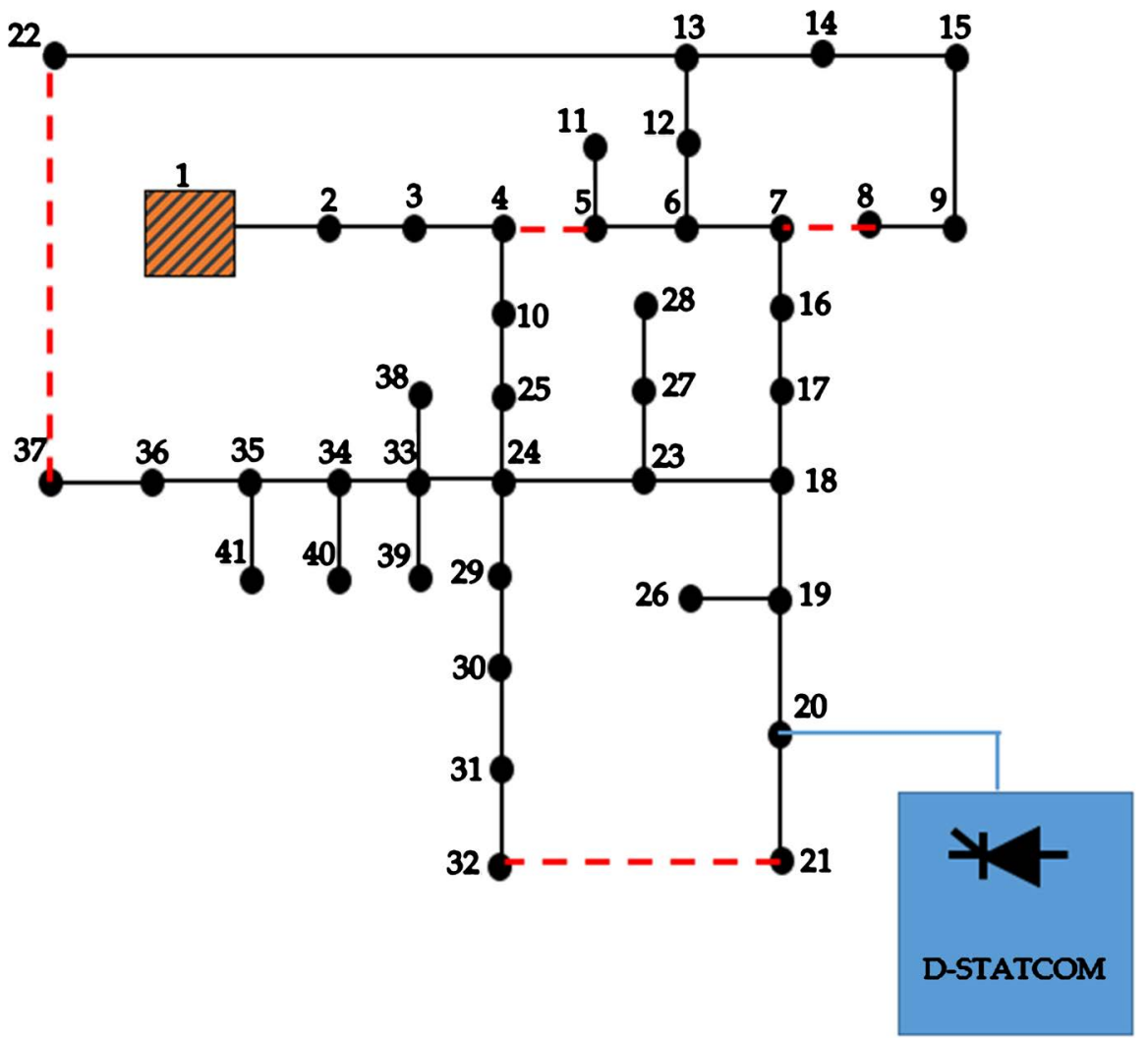

Figure 10. Network topology after both placement of DSTATCOM and reconfiguration.

configuration associated with the placement simultaneously contributes not only to qualitatively improve the technical performance of the network but also to optimize the size of DSTATCOM. This action reduces the operating costs while improving the quality of the energy supply delivered. However, the reconfiguration will have to be a period cooperation to adapt to the consumption profile and to contend with the damage of the cut-off devices.

\section{Conclusion}

In this paper, the improved ant colony algorithm is used to solve the problem of topology optimization and placement of a DSTATCOM in a real distribution network. The criteria are loss reduction, stability and voltage profiles improvements. From the results obtained, it can be concluded that the reconfiguration and placement of DSTATCOM in a distribution network contribute to reinforce the transit capacities of network equipment such as lines and transformers. This hybridization of DSTATCOM placement and reconfiguration is also the efficiency means to significantly reinforce the technical performance of the network like reducing the network losses and the power of DSTATCOM. It can then be concluded that the reconfiguration associated with the optimal placement of DSTATCOM in a distribution network makes it possible to optimize the active and reactive powers in a distribution network. For a very small effort, reconfiguration and placement improve the operational 
safety of an electrical system. The quality of solutions obtained can reassure utilities to use this tool to optimize the operation of their electrical system. The DFACTS placement associated with the reconfiguration further distances the operating point of the network from the bifurcation point.

\section{Conflicts of Interest}

The authors declare no conflicts of interest regarding the publication of this paper.

\section{References}

[1] Pérez-Arriaga, I.J. (2013) Regulation of the Power Sector. Springer Verlag, London.

[2] DD/SBEE (2017) Rapport statistique d'incident au titre de l'année 2017 réseau HTA période, Janvier à décembre.

[3] Priyadarshini, R., Prakash, R. and Priyadarshini, R. (2016) Capacitor Placement and Network Reconfiguration Simultaneously for Loss Reduction in Radial Distribution System. Indian Journal of Science and Technology, 9. https://doi.org/10.17485/ijst/2016/v9i45/104385

[4] Pinthurat, W., Pandel, A. and Ongsakul, W. (2018) Optimal Distributed Placement and Network Reconfiguration Using Hybrid Algorithm. Pathumwan Academic Journal, 8.

[5] Tolabi, H.B., Ali, M.H. and Rizwan, M. (2014) Simultaeous Reconfiguration Optimal Placement of DSTATCOM and Photovoltaic Array in a Distribution System Based on Fuzzy-ACO Approach. IEEE Transactions on Sustainable Energy, 6, 210-218. https://doi.org/10.1109/TSTE.2014.2364230

[6] Venkateswarlu, A.N., Tulasi Ra, S.S. and Sangameswara Raju, P. (2013) Impact of SVC and DG on Voltage Stability Constrained Available Transfer Capability. International Journal of Engineering Trends and Technology, 4, 3040-3044.

[7] Sugimoto, J., Yokoyama, R., Fujita, G. and Fukuyama, Y. (2006) Cooperative Allocation of SVR and SVC for Voltage Fluctuation in Case of Connecting Distributed Generators. Electrical Engineering in JAPAN, 164, 10-17.

https://doi.org/10.1002/eej.20639

[8] Tayyab, M.A. (2015) Distributed Generation Effects on Voltage Profile of Distribution Grid with SVC and Smart Inverter. IEEE Power \& Energy Society General Meeting, Denver, 26-30 July 2015.

[9] Yuvaraj, T., Devabalaji, K.R. and Ravi, K. (2015) Optimal Placement and Sizing of DSTATCOM Using Harmony Search Algorithm. Energy Procedia, 79, 759-765. https://doi.org/10.1016/j.egypro.2015.11.563

[10] Singh, J.G., Thakurta, P.G. and Soder, L. (2014) Load Curtailment Minimization by Optimal Placements of SVC/STATCOM. International Transactions on Electrical Energy Systems, 25, 2769-2780. https://doi.org/10.1002/etep.1990

[11] Oloulade, A., Imano, A.M., Vianou, A. and Tamadaho, H. (2018) Optimisation multi-critre du placement dun D-STATCOM dans un réseau de Distribution par les Colonies de Fourmis. Symposium de Genie Electrique (SGE 2018), Nancy, July 2018, 8 .

[12] Gupta, A.R. and Kumar, A. (2015) Energy Saving Using D-STATCOM Placement in Radial Distribution System under Reconfigured Network. 5th International Conference on Advances in Energy Research, Mumbai, 15-17 December 2015.

[13] Oloulade et al. (2016) Badarou Contribution à l'étude de la répartition de puissance 
et à l'évaluation des pertes dans les réseaux de transport et de distribution de la communauté électrique du Benin et de la société béninoise d'énergie électrique (CEB-SBEE), Sciences. Technologies et Développement, Edition spéciale, 87-90.

[14] Oloulade, A., Imano, A.M., Vianou, A. and Tamadaho, H. (2018) Optimization of the Number, Size and Placement of D-STATCOM in Radial Distribution Network Using Ant Colony Algorithm. American Journal of Engineering Research and Reviews, 1, 12. 